\title{
A biosynthetic alternative to human donor tissue for inducing corneal regeneration: 24- month follow-up of a phase 1 clinical study
}

Per Fagerholm, Neil S Lagali, Kimberley Merrett, W Bruce Jackson, Rejean Munger, Yuwen Liu, James W Polarek, Monica Söderqvist and May Griffith

\section{Linköping University Post Print}

N.B.: When citing this work, cite the original article.

Original Publication:

Per Fagerholm, Neil S Lagali, Kimberley Merrett, W Bruce Jackson, Rejean Munger, Yuwen Liu, James W Polarek, Monica Söderqvist and May Griffith, A biosynthetic alternative to human donor tissue for inducing corneal regeneration: 24-month follow-up of a phase 1 clinical study, 2010, Science translational medicine, (2), 46, 46-61. http://dx.doi.org/10.1126/scitranslmed.3001022

Copyright: American Association for the Advancement of Science http://www.aaas.org/

Postprint available at: Linköping University Electronic Press http://urn.kb.se/resolve?urn=urn:nbn:se:liu:diva-58809 


\title{
A Biosynthetic Alternative to Human Donor Tissue for Inducing Corneal Regeneration: 24 Month Follow-Up of a Phase I Clinical Study
}

Per Fagerholm ${ }^{1, *}$, Neil S. Lagali ${ }^{1, *}$, Kimberley Merrett ${ }^{2}$, W. Bruce Jackson ${ }^{2}$, Rejean Munger $^{2}$, Yuwen Liu ${ }^{3}$, James W. Polarek ${ }^{4}$, Monica Söderqvist ${ }^{5}$, and May Griffith ${ }^{1,2}$

* Equal contributions

${ }^{1}$ Departments of Clinical and Experimental Medicine, and Ophthalmology, Faculty of Health Sciences, Linköping University, SE-581 83 Linköping, Sweden

${ }^{2}$ University of Ottawa Eye Institute, Ottawa, Ontario K1H 8L6, Canada

${ }^{3}$ CooperVision, Inc. 5870 Stoneridge Drive, Suite 1, Pleasanton, CA 94588, USA

${ }^{4}$ FibroGen, Inc. 409 Illinois Street, San Francisco, CA, USA

${ }^{5}$ Synsam Opticians, Box 362, Trädgårdstorget 2, 58103 Linköping, Sweden

\section{One-Sentence Summary:}

A biosynthetic cornea stably integrates with host tissues 2 years after implantation and produces nerve regeneration and vision improvement.

\author{
Corresponding Author: \\ Dr. May Griffith \\ Dept. of Clinical and Experimental Medicine \\ Faculty of Health Sciences \\ Linköping University \\ Cell Biology Building, Level 10 \\ 58183 Linköping \\ Sweden \\ Tel: 46-(0)13 224254 \\ E-mail: May.Griffith@liu.se
}




\begin{abstract}
Corneas from human donors are used to replace damaged tissue and treat corneal blindness, but there is a severe worldwide shortage of donor corneas. We conducted a Phase I clinical study in which biosynthetic mimics of corneal extracellular matrix were implanted to replace the pathologic anterior cornea of ten patients with significant vision loss, with the aim of facilitating endogenous tissue regeneration without the use of human donor tissue. The biosynthetic implants remained stably integrated and avascular 24 months after surgery, without the need for long-term use of the steroid immunosuppression that is required for traditional allotransplantation. Corneal reepithelialization occurred in all patients, although a delay in epithelial closure as a result of the overlying retaining sutures led to early, localized implant thinning and fibrosis in some patients. The tear film was restored, and stromal cells were recruited into the implant in all patients. Nerve regeneration was also observed and touch sensitivity was restored, both to an equal or greater degree than is seen with human donor tissue. Vision at 24 months improved from preoperative values in six patients. With further optimization, biosynthetic corneal implants could offer a safe and effective alternative to the implantation of human tissue to help address the current donor cornea shortage.
\end{abstract}




\section{INTRODUCTION}

The human cornea is the transparent outermost surface of the eye and major refractive element of the visual system; its function depends upon its optical clarity. Irreversible loss of optical quality of the cornea due to disease or damage results in permanent vision loss or blindness. The World Health Organization (WHO) estimates that 50 million people worldwide are bilaterally blind, and at least 150 million people have impaired vision in both eyes $(1,2)$. Although cataracts are responsible in almost half of the patients with vision loss, corneal damage and disease is the next largest cause. Trachoma, an eye disease caused by the bacterium Chlamydia trachomatis, can lead to corneal inflammation and scarring, and affects roughly 5 million people worldwide. Ulceration and trauma are responsible for an additional 1.5 to 2 million new patients with corneal blindness annually (2).

The most successful and widely accepted treatment for corneal blindness worldwide is full-thickness replacement of the damaged tissue with a human donor cornea in a procedure known as penetrating keratoplasty (PK). For many conditions where the posterior-most layer (endothelium) is unaffected, both anterior lamellar keratoplasty (ALK) and deep anterior lamellar keratoplasty (DALK), which are procedures that preserve the recipient's endothelium, result in improved graft survival compared to PK. Regardless of the technique, the fundamental problem with corneal replacement is a severe shortage of donor tissue, resulting in approximately 10 million untreated patients worldwide, and 1.5 million new cases of blindness annually (2). Additionally, as for all solid organ transplants, donor-derived infection is a serious complication and a leading 
concern in eye and tissue banking (3-5). Pre-transplant screening costs are high and will escalate as more rigorous testing for an increasing number of transmissible pathogens is implemented.

Despite efforts to develop corneal substitutes to alleviate both the shortage and drawbacks of human donor tissues (6), surgery with allogeneic donor tissue has remained the gold standard for over a century. Recently, however, developments in bioengineered corneal substitutes designed to replace the full or partial thickness of damaged or diseased corneas have been reported (6,7). These range from fully synthetic prostheses (eg. keratoprostheses) made from poly-methacrylates, that aim to replace the cornea's refractive function $(6,7)$, to tissue-engineered cell-based constructs $(8)$ and hydrogels that also permit the integration of the implant and regeneration of the host tissues (9-11). At present, keratoprostheses are the only non-allogeneic option approved for human use. Although improving and well-retained, keratoprostheses still suffer from the drawbacks of a complex implantation procedure and serious complications, including retroprosthetic membrane formation, calcification, infection, glaucoma, and retinal detachment. Their use is therefore limited to cases in which allogeneic tissue has failed repeatedly or is contraindicated $(6,7,12)$.

Our goal was to adopt a regenerative medicine approach to design a primary alternative to the use of allogeneic tissue for restoring sight by corneal replacement. Specifically, we proposed to induce regeneration of the damaged corneas by implantation of an acellular matrix that serves to facilitate regeneration by emulating the functions of the highly bioactive natural extracellular matrix (ECM) scaffolding of the cornea. Because the corneal ECM is largely collagenous (13), and collagen is a biopolymer that is 
amenable to modification, we selected collagen as our starting material. The collagen was synthetically crosslinked and moulded into an implantable, biosynthetic corneal substitute (9-11). The substitute is cell-free and relies on re-population by host cells to restore corneal function, thereby avoiding the rejection reaction and the need for long-term steroid use. We have successfully implanted a range of these corneal substitutes in animals $(9,11)$. With the availability of recombinant collagen $(14,15)$, we have developed a class of implants (16), made from human materials, that alleviate the risk of transmission of infectious agents (such as viruses or prions) inherent in components from animals, while being produced under stringent conditions for quality and safety assurance. We demonstrated safety, biocompatibility, and regenerative potential of lamellar implantation of a recombinant human collagen-based material in mini-pigs $(16,17)$. Subsequently we implanted lamellar implants of this biosynthetic material in ten human subjects for keratoconus or corneal scarring and reported the early morphologic and clinical findings (10). We report here the safety, clinical efficacy, and detailed morphologic and physiologic results from the two-year follow-up of these patients, and compare these results to transplanted human corneal tissue and the response in normal eyes. We specifically evaluated the long term integration and stability of the implanted material, the effectiveness of the surgical procedure, and the degree to which the implants enabled regeneration of endogenous epithelium, nerves, stroma, and tear film. 


\section{RESULTS}

Ten patients were implanted with $500 \mu \mathrm{m}$ thick biosynthetic corneal substitutes, comprising 1-ethyl-3-(3-dimethylaminopropyl) carbodiimide (EDC)-crosslinked recombinant human collagen. These replacements were optically clear (white light transmittance of $95.1 \pm 0.5 \%$ ), with refractive index 1.35 . They were trephined to size, and implanted by anterior lamellar keratoplasty (ALK) with overlying sutures to secure in place (Fig. 1). Sutures were removed after $6.5 \pm 3$ weeks (mean \pm standard deviation; Table 1), after which immunosuppressive steroids were stopped while anti-infective medications were continued for another $5.4 \pm 3$ weeks. Overall, all implanted corneas remained free of major complications (such as stromal edema, neovascularization, or prolonged inflammation), and there were no signs of rejection within the implant or surrounding patient corneal rim at any time during the 24 month follow-up period. There were no patient complaints of pain or discomfort.

After an initial decrease, the thickness of the central cornea in implanted eyes stabilized after the third postoperative month. For the 10 patients, mean central corneal thickness at 24 months $(403 \pm 109 \mu \mathrm{m})$ was comparable to thickness measured at $3 \mathrm{~m}$ (404 $\pm 87 \mu \mathrm{m}), 6 \mathrm{~m}(420 \pm 100 \mu \mathrm{m})$, and $12 \mathrm{~m}(385 \pm 90 \mu \mathrm{m})$. At 24 months, the thinnest cornea had a central thickness of $211 \mu \mathrm{m}$ and two corneas had a central thickness of at least $550 \mu \mathrm{m}$. Intraocular pressure in all operated eyes was unaffected by the surgery and was within the normal range of $9-20 \mathrm{~mm} \mathrm{Hg}$ at 24 months. There was no blockage of aqueous humor flow within the eye, and iris angles were open as observed by anterior segment optical coherence tomography (ASOCT). One patient also underwent (unrelated) cataract surgery in the implanted eye with no adverse effects to the implant. 
Initial migration of epithelial cells over the implants was rapid, but the coverage was still incomplete by fluorescein staining one month after surgery. Close inspection revealed that epithelial migration over the implant was halted at the crossing points of sutures in the center of the implants (Fig. 2A and B). Once sutures were removed, epithelial coverage was completed in all eyes. However, discrete focal areas of haze remained in the regions initially showing delayed epithelial closure, even at 24 months (Fig. 2C, D, see also Supplementary Fig. S1, S2). Apart from these focal areas of haze, the implants remained transparent. Good overall corneal transparency at 24 months (Fig. 3) was demonstrated by the clear visualization of the retina through the implanted cornea by fundus photography (Fig. 4). At 24 months, the regenerated epithelium was morphologically normal in all patients, with good stratification and stable attachment (Fig. 5B). Tear production in the film overlying the epithelium was normal ( $>15 \mathrm{~mm}$ in 5 min, without anaesthesia) in 7 of 10 operated and 6 of 10 contralateral eyes (24 months, Table 1). Tear osmolarity in implanted eyes was not different than untreated eyes (operated, $304.7 \pm 12 \mathrm{mOsm} / \mathrm{L}$; unoperated, $305.3 \pm 22 \mathrm{mOsm} / \mathrm{L} ; 10$ eyes). The threshold value of $316 \mathrm{mOsm} / \mathrm{L}$, indicative of clinically dry eye (19), was exceeded in one patient (Patient 3) for both operated and unoperated eyes. Within the first six months after surgery, the implants were firmly integrated within the patient's eye, and corresponding in vivo confocal microscopy (IVCM) of the implant-recipient interfaces showed highly reflective areas that were consistent with activated stromal cells migrating into the implant and anchoring it in place (10). At 24 months, in 7 of 10 patients IVCM revealed distinct, discrete nuclei of stromal cells within the initially acellular implant (Fig. 5H), with an appearance consistent with quiescent keratocytes found in the corneal stroma of 
normal, unoperated corneas (Fig 5G). Stromal cell migration from the recipient into the central implant appeared to be gradual, and the extent of repopulation of the initially acellular implant varied locally within the implant, and also from patient to patient (Supplementary Fig. S3).

Regenerating nerves were first noted at the basal epithelium between three and six months after surgery in 6 of 10 implanted corneas. Beaded fibers, which typify the subbasal epithelial nerve plexus, started appearing at 12 months in four patients. A substantial increase in the density of sub-basal nerves was found between 12 to 24 months by IVCM image analysis (Table 2).

Nerve function was assessed by measuring corneal sensitivity to mechanical touch with a Cochet-Bonnet aesthesiometer. Some corneal sensitivity returned within the first 12 months after surgery in all 10 eyes $(25 \pm 14 \mathrm{~mm})$ and improved in all by 24 months (35 $\pm 17 \mathrm{~mm}$ ). Sensitivity, however, remained below the response of contralateral eyes with intact innervation that showed an average sensitivity of $60 \mathrm{~mm}(60 \pm 0 \mathrm{~mm})$ corresponding to a normal level (Fig. 6). Four of the ten implanted corneas, however, had a sensitivity of $50 \mathrm{~mm}$ or better by 24 months.

At 24 months, best spectacle-corrected visual acuity (BSCVA) improved from the preoperative value in 6 patients, remained unchanged in 2 patients, and decreased in 2 patients (Table 1). BSCVA in the implanted eyes was significantly lower than the values seen in a population of 60 keratoconus patients with penetrating donor tissue transplants two years after surgery $(20)(\mathrm{P}<0.001$; Mann-Whitney $\mathrm{U}$ test $)$ (Table 3). However, when patients with biosynthetic implants were fitted with rigid, gas-permeable contact lenses, their 24 month BCLVA was comparable to 24 month BSCVA in the transplanted 
keratoconus population $(\mathrm{P}=0.55$, Table 3$)$. Although all patients who received the biosynthetic implants were contact lens intolerant before surgery (i.e., could not wear contact lenses for more than 2 hours per day without significant irritation), 6 of 7 patients tested could tolerate the rigid contact lenses at the time of the 24 month follow-up. 


\section{DISCUSSION}

The human cornea is a transparent, hydrated collagenous extracellular matrix $(80 \%$ water, $13.6 \%$ collagen, $0.9 \%$ glycosaminoglycans) containing specialized fibroblast-like cells in a stromal layer, sandwiched between an outermost, stratified epithelium and an innermost endothelial layer (13). Biosynthetic corneal substitutes, based on simple mimics of the natural human extracellular matrix (such as those used in this study), have been tested as microenvironments in which to induce regeneration of tissues and organs that do not regenerate spontaneously (21). EDC is one of a family of protein crosslinking reagents, the water soluble carbodiimides (WSCs), which have a unique property: the reagents themselves do not become incorporated into the hydrated matrix as part of the final cross-links, so there is no possibility of toxic substances being released into tissues from cross-link breakdown after implantation into patients (22).

We have reported here that the first ten patients to receive such biosynthetic, collagen-based mimics as partial thickness corneal implants have retained the implant for two years without rejection, peripheral or central vascularization, or infection, and without the need for long-term steroid immunosuppression. In all patients, a stable, morphologically normal epithelium covered the implant and established a viable ocular surface that supported an adequate tear film and restoration of mechanical touch sensitivity. Rapid host-implant integration enabled suture removal much earlier than the typical 4 to 8 months after ALK with human tissue $(23,24)$, with implants being stably anchored to the surrounding cornea by recipient stromal cell infiltration at the interfaces.

Additionally, these implants support human nerve regeneration. Nerve regeneration has not been previously possible in corneal prosthetics $(6,7,12)$, nor has it been described 
in any other alternative corneal substitute tested in humans. Sub-basal epithelial nerves regenerated in nine of ten patients receiving implants and increased in density during the first two years after surgery. In other studies, nerve regeneration has been reported to be slower, occurring as late as 10 years after lamellar keratoplasty in some patients (25). Corneal nerves, situated in the anterior two-thirds of the stroma, are transected in ALK and PK procedures. Previous IVCM studies show a complete absence of sub-basal nerves in a high proportion of grafts up to 31 years after PK (26-29), and contact aesthesiometry studies have revealed that grafts remain anaesthetic up to 32 years after PK $(26,30,31)$. Our observations of regenerated sub-basal nerves and return of ocular surface sensitivity within 24 months of surgery suggest that biosynthetic implants may facilitate rapid nerve regeneration. Nerves are essential for the protective aversion response of the cornea to external stimuli, and play a vital role in the maintenance of epithelial health and integrity, in corneal wound healing, and in regulating secretion of the preocular tear film (32).

In seven patients, discrete focal areas of haze with thinning were observed in areas where the central suture passed over the implant, suggesting that tight retaining sutures overlying the implant delayed central epithelialization of some implants, creating an epithelial defect. This delay likely initiated an early inflammatory response, followed by induction of metalloproteinase enzymes and subsequent localized implant thinning and early stromal invasion by repair fibroblasts. The sutures most likely also accounted for the localized thinning and hence the surface irregularity observed. When this surface irregularity, observed on slit lamp examination (Fig. 2) was compensated for by placement of rigid contact lenses, vision substantially improved. Thus the method of implant retention is an important consideration in the implantation of biosynthetic corneal 
substitutes. The employment of suture-free retention methods (eg., host-donor shaping with a femtosecond laser (33) or tissue adhesives (34)) would be expected to improve visual outcome. A revised surgical method (eg., using interrupted sutures), as well as the use of a biosynthetic implant with higher tensile strength and greater resistance to enzymatic degradation, may be necessary to retain a smooth anterior curvature and prevent early implant thinning, particularly in keratoconus patients with active disease. Such newer biosynthetic implants, developed by our group, have shown favorable results after 12 months in mini-pigs (35) and are being further optimized for clinical application.

The haze created at the interface between the stroma and the implant was likely an additional source of reduced vision in patients with implants. This haze indicating wound repair at the implant-to-recipient interface, was visible on ASOCT scans, and is known to occur in ALK as well (36). Resection of the entire stromal thickness, as in deep anterior lamellar keratoplasty (DALK), limits the formation of this interface, and is therefore becoming the procedure of choice for conditions in which the corneal endothelium is not affected, such as keratoconus and stromal scars $(36,37)$. Although in this Phase I study we chose ALK as an initial, safer procedure to evaluate the integration of biosynthetic implants into patient corneas, improved visual outcomes in DALK relative to ALK (36) may warrant its adoption in future studies that test efficacy. The delay in vision improvement in patients with implants noted in this study, however, is similar to effects described with human donor tissue implanted by ALK. In a 19-year retrospective study of ALK patients (38), the four most significant post-operative vision-limiting factors noted were graft-host interface haze, graft surface irregularities and/or astigmatism, graft stromal haze, and opacifications caused by delay of epithelial closure. 
Despite the need to refine the surgical technique for optimum visual outcome, the implants in this study resulted in overall improved vision, while demonstrating key safety, biocompatibility, and regenerative features needed from a biosynthetic implant that would enable its use as a viable substitute for human donor tissue in therapeutic applications. Implants were accepted by the host with only minimal use of steroids for initial prophylactic immunosuppression, became epithelialized, innervated, populated with cells, integrated into host tissue, and supported normal tear production and return of touch sensitivity. Additionally, unlike keratoprostheses, for which post-operative glaucoma is a major complication (39), these implants did not result in any intraocular pressure change. The biosynthetic implant thus enabled regenerative repair of resected corneal tissue without the use of animal-derived or potentially toxic components.

A larger number of patients, a wider range of clinical indications, modified surgical techniques, and continued biomaterials development are necessary to determine the full potential of biosynthetic corneal substitutes for relieving the burden of organ donation and corneal blindness. Nevertheless, our results demonstrate that biosynthetic corneas promoting endogenous regeneration are a possible alternative to human donor tissue for implantation in conditions where the endothelium is uncompromised. 


\section{MATERIALS AND METHODS}

\section{Patients}

After approval from the Swedish Medical Products Agency and the Regional Ethical Review Board in Linköping, Sweden (application no. M205-06), trial registration (EudraCT no. 2006-006585-42), and with informed consent after the nature and potential risks of the procedure was explained, ten patients aged 18 to 75 years were enrolled in a Phase I clinical trial and implanted with biosynthetic corneal substitutes. Patients were consecutive cases meeting the following criteria: on the waiting list for a first corneal transplantation, having a clear posterior stroma and a normal endothelium, and with a non-scarred, avascular peripheral cornea. Nine of ten eyes had a diagnosis of advanced keratoconus and were contact lens intolerant. The tenth eye had a permanent mid-stromal scar in the visual axis secondary to bacterial keratitis. The indication for transplantation in all cases was improvement of visual acuity. All patients were operated on by a single surgeon (PF) between October and November 2007 at Linköping University Hospital and were followed for 24 months after surgery.

\section{Corneal Matrix Substitutes}

Clinical-grade recombinant human collagen, type III (RHCIII) made in yeast (Pichia pastoris) (FibroGen Inc.) was freeze-dried and reconstituted. A $10 \% \mathrm{wt} / \mathrm{wt}$ aqueous RHCIII solution was mixed with a water-soluble chemical crosslinking agent [1-ethyl-3(3-dimethylaminopropyl) carbodiimide (EDC)] and its co-reactant [Nhydroxysuccinimide (NHS)], in a $0.4: 1$ EDC to collagen ratio at $4^{\circ} \mathrm{C}$. The mixture was 
immediately dispensed into curved polypropylene moulds $(500 \mu \mathrm{m}$ thick, $12 \mathrm{~mm}$ diameter) and cured in $100 \%$ humidity at $21^{\circ} \mathrm{C}$ for 24 hours. The cured implants were removed from moulds in sterile phosphate buffered saline (PBS), washed three times in sterile PBS, and stored in a PBS solution containing $1 \%$ chloroform to maintain sterility. A batch of sixteen clinical-grade samples was produced in a laboratory certified to follow good manufacturing practices. Ten samples were used for patient implantation and the other six underwent laboratory characterization as described (16), which consisted of measurement of optical transmittance (380-780nm band, Lambda 25 UV/VIS spectrometer, Perkin Elmer Inc.) and refractive index (at $21^{\circ} \mathrm{C}$ with bromonaphthalene as the calibration agent, Model C10 Abbe refractometer, VEE GEE Scientific Inc.).

\section{Procedures}

Anterior lamellar keratoplasty (ALK) was performed on patients under either local or general anaesthesia. The patient's cornea was first cut with a 6.0 to $6.5 \mathrm{~mm}$ diameter trephine set to a depth of $200 \mu \mathrm{m}$ (Baron). The incision was then deepened manually with a diamond knife set to a depth of 370 to $400 \mu \mathrm{m}$. Manual lamellar dissection was then used to remove the pathologic corneal tissue. A punch trephine (Baron) was used to cut the biosynthetic implant, producing a final lamellar implant $500 \mu \mathrm{m}$ thick and $0.25 \mathrm{~mm}$ larger in diameter than that of the recipient corneal bed. Once in place, the implant was anchored with three to four overlying 10-0 nylon mattress sutures to avoid puncturing the implant material. Two to three antibiotic eye drops (chloramphenicol 0.5\%, MINIMS Chauvin Pharmaceuticals) were given at the end of surgery, and an inert bandage contact lens of $72 \mathrm{~mm}$ diameter (Starsoft 72, Star Lens AB, 76.5\% water/23.5\% poly-xylon D 
with UVR blocker) was placed on the eye. Patients received one drop each of chloramphenicol and Opnol topical steroid drops (0.1\% dexamethasone without preservatives, Clean Chemical Sweden) three times daily until the contact lens and sutures were removed. After suture removal, patients received either Chloromycetin eye ointment (chloramphenicol 1\%, Pfizer), applied 2 to 3 times daily, or Terracortil with Polymyxin B eye drops (oxytetracycline $0.5 \%$, hydrocortisone acetate $1.5 \%$, and polymyxin B sulphate 10,000 units, Pfizer) applied 1 to 2 drops daily.

\section{Clinical Evaluation}

Post-operative follow-up occurred at day 1, 3, 6, week 2, 3, 4, 6, and month 2, 3, 4, 5, 6, 9, 12, 18 and 24. At each follow-up visit, the eye was examined with a slit lamp for signs of infection or inflammation. Detailed follow-up was performed at 3, 6, 9, 12, 18 and 24 months. We assessed: central corneal thickness (ultrasound pachymeter; Tomey SP-2000), intraocular pressure (Goldmann applanation tonometer, Haag-Streit), aqueous tear film production (Schirmer test without anaesthesia), dry eye (TearLab Osmolarity System; Tear Lab), topography (Orbscan II; Bausch \& Lomb), standard manifest refraction, and best corrected visual acuity (Monoyer letter chart) with spectacles (BSCVA) or gas permeable rigid contact lens (BCLVA). Additionally, corneal status was documented visually by slit lamp photography, anterior segment optical coherence tomography (ASOCT; Visante, Carl Zeiss Meditec), and in vivo confocal microscopy (IVCM; HRT3-RCM, Heidelberg Engineering).

Corneal nerve function (corneal touch sensitivity) was assessed by Cochet-Bonnet contact aesthesiometry (Cochet-Bonnet aesthesiometer; Luneau Ophthalmologie), where 
a nylon filament probing the ocular surface was progressively reduced from $60 \mathrm{~mm}$ (normal sensitivity) to $10 \mathrm{~mm}$ (minimal sensitivity) to elicit a response. Filament length at initial response was recorded. Corneal nerve regeneration was assessed with IVCM (17), by manually scanning the basal epithelial plane to visualize sub-basal nerve fiber bundles, first in the central 2 to $3 \mathrm{~mm}$ and then in the mid-peripheral 3 to $5 \mathrm{~mm}$ of cornea. Images were digitally captured at 8 frames/sec during scanning. A single corneal image with the greatest density of central sub-basal nerves was selected from each patient at each follow-up time, for further quantitative analysis. Nerve density (expressed in microns of total nerve length in a digital frame per frame area in $\mathrm{mm}^{2}$ ) was quantified from images using nerve tracing methodologies to determine total nerve length in a frame, as described in detail elsewhere (17). Two independent observers traced each image in a blinded manner, and the nerve density for a given image was taken as the mean value. The $95 \%$ inter-observer limits of agreement for nerve density were calculated from inter-observer differences at each follow-up time using the Bland-Altman method (18).

\section{Assessment of clinical efficacy}

To gauge the efficacy of the biosynthetic implant for vision restoration, the study protocol included a comparison of visual acuity in study patients with the two-year postoperative vision in a population of keratoconus patients who received full-thickness human donor corneas by penetrating keratoplasty (the most common treatment for the condition). Data for the keratoconus patient population was extracted from the Swedish Corneal Transplant Register, for patients operated in the calendar year 2005 (20) and a t- 
test was used to compare visual outcome. Statistical analysis was performed with SigmaStat 3.5 (Systat Software Inc.), with a two-tailed test and P $<0.05$ considered significant.

\section{SUPPLEMENTARY MATERIAL}

Fig. S1. Slit lamp biomicroscopy showing the time course of epithelialization in Patient 3.

Fig. S2. Slit lamp biomicroscopy showing the time course of epithelialization in Patient 6.

Fig. S3. Stromal cell migration into the biosynthetic implant at 24 months in four patients. 


\section{REFERENCES AND NOTES}

1. A. Foster, Vision 2020--the Right to Sight. Trop. Doct. 33, 193-194 (2003).

2. J. P. Whitcher, M. Srinivasan, M. P. Upadhyay, Corneal blindness: a global perspective. Bull. World Health Organ. 79,214-221 (2001).

3. D. O'Day, Diseases potentially transmitted through corneal transplantation. Ophthalmology 96, 1133-1137 (1989).

4. L. Remeijer, J. Maetzdorf, P. Doornenbal, G. M. G. M. Verjans, A. D. M. E. Osterhaus, Herpes simplex virus 1 transmission through corneal transplantation. Lancet 357, 442 (2001).

5. S. S. Hassan, K. R. Wilhemus, P. Dahl, G. C. Davis, R. T. Roberts, K. W. Ross, B. H. Varnum et al, Infectious disease risk factors of corneal donor grafts. Arch. Ophthalmol. 126, 235-239 (2008).

6. D. Myung, P. E. Duhamel, J. R. Cochran, J. Noolandi, C. N. Ta, C. W. Frank, Development of hydrogel-based keratoprostheses: A materials perspective. Biotechnol. Prog. 24,735-741 (2008).

7. M. A. Princz, H. Sheardown, M. Griffith, Corneal tissue engineering vs synthetic artificial corneas. In: Biomaterials and Regenerative Medicine in Ophthalmology. TV Chirila, Ed. CRC Press, Woodhead Publ. Ltd., Cambridge, UK (2009).

8. P. Carrier, A. Deschambeault, M. Talbot, C. J. Giasson, F. A. Auger, S. L. Guerin, L. Germain, Characterization of wound reepithelialization using a new human tissueengineered corneal wound healing model. Invest. Ophthalmol. Vis. Sci. 49, 1376-85 (2008).

9. F. Li, D. J. Carlsson, C. P. Lohmann, E. J. Suuronen, S. Vascotto, K. Kobuch, H. Sheardown, R. Munger, M. Griffith, Cellular and nerve regeneration within a biosynthetic extracellular matrix: corneal implantation. Proc. Natl. Acad. Sci. USA 100, 15346-15351 (2003).

10. P. Fagerholm, N. Lagali, D. J. Carlson, K. Merrett, M. Griffith, Corneal regeneration following implantation of a biomimetic tissue-engineered substitute. Clin. Transl. Sci. 2, 162-164 (2009).

11. C. R. McLaughlin, M. C. Acosta, C. Luna, W. Liu, C. Belmonte, M. Griffith, J. Gallar, Regeneration of functional nerves within full thickness collagen-phosphorylcholine corneal substitute implants in guinea pigs. Biomaterials 31, 2770-2778 (2010).

12. A. J. Aldave, K. M. Kamal, R. C. Vo, F. Yu, The Boston type I keratoprosthesis. Improving outcomes and expanding indications. Ophthalmology 116, 640-651 (2009). 
13. T. Nishida, Cornea: Fundamentals of cornea and external disease in Cornea (J. J. Krachmer, M. J. Mannis, E.J. Holland, Eds. (Mosby-Year Book Inc., St. Louis, Missouri, 1997), pp.3-26).

14. D. Olsen, C. Yang, M. Bodo, R. Chang, S. Leigh, J. Baez, D. Carmichael, M. Perala, E.R. Hamalainen, M. Jarvinen, J. Polarek, Recombinant collagen and gelatin for drug delivery. Adv. Drug. Deliv. Rev. 55, 1547-1567 (2003).

15. C. Yang, J. Patrick, J. Baez, M. Nokelainen, J. Balan, J. Tang, R. Spiro, J. W. Polarek, The application of recombinant human collagen in tissue engineering. BioDrugs 18, 103119 (2004).

16. K. Merrett, P. Fagerholm, C. R. McLaughlin, S. Dravida, N. Lagali, N. Shinozaki, M. A. Watsky, R. Munger, Y. Kato, F. Li, C. J. Marmo, M. Griffith, Tissue engineered recombinant human collagen-based corneal substitutes for implantation: performance of type I versus type III collagen. Invest. Ophthalmol. Vis. Sci. 49, 3887-3894 (2008).

17. N. Lagali, M. Griffith, P. Fagerholm, K. Merrett, M. Huynh, R. Munger, Innervation of tissue-engineered recombinant human collagen-based corneal substitutes: a comparative in vivo confocal microscopy study. Invest. Ophthalmol. Vis. Sci. 49, 3895-3902 (2008).

18. D. G. Altman, J. M. Bland, Statistical methods for assessing agreement between two methods of clinical measurements. Lancet 327, 307-310 (1986).

19. A. Tomlinson, S. Khanal, K. Ramaesh, C. Diaper, A. McFadyen, Tear film osmolarity: determination of a referent for dry eye diagnosis. Invest. Ophthalmol. Vis. Sci. 47, 43094315 (2006).

20. M. Claesson, W. J. Armitage, P. Fagerholm, U. Stenevi, Visual outcome in corneal grafts: a preliminary analysis of the Swedish Corneal Transplant Register. Br. J. Ophthalmol. 86, 174-180 (2002).

21. I. V. Yannas, Biologically active scaffolds based on collagen-GAG copolymers in Scaffolding in Tissue Engineering (PX Ma, J Elisseeff Eds. (CRC Press, Boca Raton, FL, USA, 2006), pp. 3-12).

22. P. F Gratzer, J. M. Lee, Control of $\mathrm{pH}$ alters the type of cross-linking produced by 1ethyl-3-(3-imethylaminopropyl)-carbodiimide (EDC) treatment of acellular matrix vascular grafts. J. Biomed. Mater. Res. (Appl. Biomater.). 58, 172-179 (2001).

23. I. Bahar, I. Kaiserman, S. Srinivasan, J. Ya-Ping, A. R. Slomovic, D. S. Rootman, Comparison of three different techniques of corneal transplantation for keratoconus. Am. J. Ophthalmol. 146, 905-912 (2008). 
24. G. Marchini, L. Mastropasqua, E. Pedrotti, M. Nubile, M. Ciancaglini, A. Sbabo, Deep lamellar keratoplasty by intracorneal dissection. A prospective clinical and confocal microscopic study. Ophthalmology 113, 1289-1300 (2006).

25. S. L. Kaminski, R. Biowski, J. R. Lukas, D. Koyuncu, G. Grabner, Corneal sensitivity 10 years after epikeratoplasty. J. Refract. Surg. 18, 731-736 (2002).

26. A. Richter, C. Slowik, S. Somodi, H. P. Vick, R. Guthoff, Corneal reinnervation following penetrating keratoplasty - correlation of esthesiometry and confocal microscopy. Ger. J. Ophthalmol. 5,513-517 (1997).

27. J. G. Hollingsworth, N. Efron, A. B. Tullo, A longitudinal case series investigating cellular changes to the transplanted cornea using confocal microscopy. Cont. Lens. Ant. Eye 29,135-141 (2006).

28. T. Darwish, A. Brahma, N. Efron, C. O'Donnell, Sub-basal nerve regeneration after penetrating keratoplasty. Cornea 26, 935-940 (2007).

29. S. V. Patel, J. C. Erie, J. W. McLaren, W. M. Bourne, Keratocyte density and recovery of sub-basal nerves after penetrating keratoplasty and in late endothelial failure. Arch. Ophthalmol. 125, 1693-1698 (2007).

30. G. N. Rao, T. John, N. Ishida, J. V. Aquavella, Recovery of corneal sensitivity in grafts following penetrating keratoplasty. Ophthalmology 92, 1408-1411 (1985).

31. W. D. Mathers, J. V. Jester, M. A. Lemp, Return of human corneal sensitivity after penetrating keratoplasty. Arch. Ophthalmol. 106, 210-211 (1988).

32. L. J. Müller, C. F. Marfurt, F. Kruse, T. M. T. Tervo, Corneal nerves: structure, contents and function. Exp. Eye Res. 76,521-542 (2003).

33. S. H. Yoo, G. D. Kymionis, A. Koreishi, T. Ide, D. Goldman, C. L. Karp, T. P. O'Brien, W. W. Culbertson, E. C. Alfonso, Femtosecond laser-assisted sutureless anterior lamellar keratoplasty. Ophthalmology 115, 1303-1307 (2008).

34. Y. M. Por, Y. L. Tan, J. S. Mehta, D. T. H. Tan, Intracameral fibrin tissue sealant as an adjunct in tectonic lamellar keratoplasty for large corneal perforations. Cornea 28, 451455 (2009).

35. W. Liu, C. Deng, C. R. McLaughlin, P. Fagerholm, M. A. Watsky, B. Heyne, J. C. Scaiano, N. S. Lagali, R. Munger, F. Li, M. Griffith, Collagen-phosphorylcholine interpenetrating network hydrogels as corneal substitutes. Biomaterials 30, 1551-1559 (2009). 
36. N. Ardjomand, S. Hau, J. C. McAlister, C. Bunce, D. Galaretta, S. J. Tuft, D. F. P. Larkin, Quality of vision and graft thickness in deep anterior lamellar and penetrating corneal allografts. Am. J. Ophthalmol. 143, 228-235 (2007).

37. C. L. Funnell, J. Ball, B. A. Noble, Comparative cohort study of the outcomes of deep lamellar keratoplasty and penetrating keratoplasty for keratoconus. Eye 20,527-532 (2006).

38. H. K. Soong, D. G. Katz, A. A. Farjo, A. Sugar, R. F. Meyer, Central lamellar keratoplasty for optical indications. Cornea 18, 249-256 (1999).

39. K. Hille, A. Hille, K. W. Ruprecht, Medium term results in keratoprostheses with biocompatible and biological haptic. Graefe's. Arch. Clin. Exp. Ophthalmol. 244, 696704 (2006).

40. R. L. Niederer, D. Perumal, T. Sherwin, C. N. J. McGhee, Corneal innervation and cellular changes after corneal transplantation: an in vivo confocal microscopy study. Invest. Ophthalmol. Vis. Sci. 48, 621-626 (2007).

41. Acknowledgements: We thank our many past and present collaborators, in particular: D. J. Carlsson, F. Li, C. P. Lohmann, K. Kobuch, M. Watsky, N. Shinozaki, Y. Kato, D. Priest and C. R. McLaughlin. Funding: This study was supported by grants from the Swedish Research Council and County of Östergötland to PF, an EU Marie Curie International Fellowship to NL, and the Canadian Stem Cell Network to MG. Author contributions: PF performed surgeries, clinical follow-up, and clinical data collection, assisted in study design, data analysis and interpretation, and critical revision of the manuscript. NL assisted in study design, clinical data collection, data analysis and interpretation, drafted the manuscript, and assisted in critical revision of the manuscript. KM developed the process of formulating the biosynthetic implants and assisted in manuscript revision. WBJ assisted in study design, clinical data collection, data interpretation and critical revision of the manuscript. RM assisted in data analysis and interpretation, and critical manuscript revision. YL fabricated the implants used in this 
study and assisted in manuscript revision. JP developed the recombinant human collagen used in this study and assisted in manuscript revision. MS assisted in postoperative clinical data collection, performed custom contact lens fitting in patients to achieve BCLVA, and assisted in manuscript revision. MG conceived the concept of endogenous corneal regeneration within a biosynthetic implant, oversaw development and production of the biosynthetic implants, participated in study design, data analysis and interpretation, and manuscript preparation and critical revision. Competing interests: J.W. Polarek is Vice President for Protein Therapeutics and Collagen Development at FibroGen, Inc. and is compensated in part for his activities aimed at the development of recombinant human collagen for medical use. Patents relating to the production of recombinant human type III collagen used in the biosynthetic corneal implant described in this study are owned by FibroGen, Inc. Recombinant human type III collagen materials are available under license from FibroGen, Inc. None of the other authors had any conflict of interest from the time of recruitment and selection of patients to the time of final manuscript submission. Although Cooper Vision GMP laboratories were used to fabricate the biosynthetic implants, the company has no commercial interest in the technology. A patent application related to the biomaterials formulation described in this study has been filed and assigned to the Ottawa Hospital Research Institute (OHRI), and is currently licensed to Eyegenix, Inc.; a wholly-owned subsidiary of Cellular Bioengineering, Inc., Hawaii for use in the field of corneal transplantation. An existing OHRI-LV Prasad Eye Institute, India memorandum of understanding allows for fabrication and use of the biosynthetic implants for transplantation on a non-commercial basis. 


\section{FIGURE AND TABLE CAPTIONS}
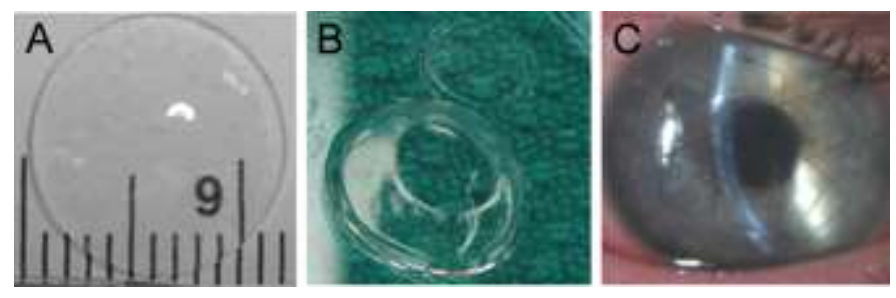

Figure 1. Fabricated cornea and implantation method. (A) An example of optically clear, biosynthetic corneal substitutes used in these studies. (B) These were trephined to prepare a button for corneal implantation. Damaged host tissue was removed to a similar depth and diameter, and replaced by this button. (C) After implantation, the button was held in place with three overlying 10-0 mattress sutures.
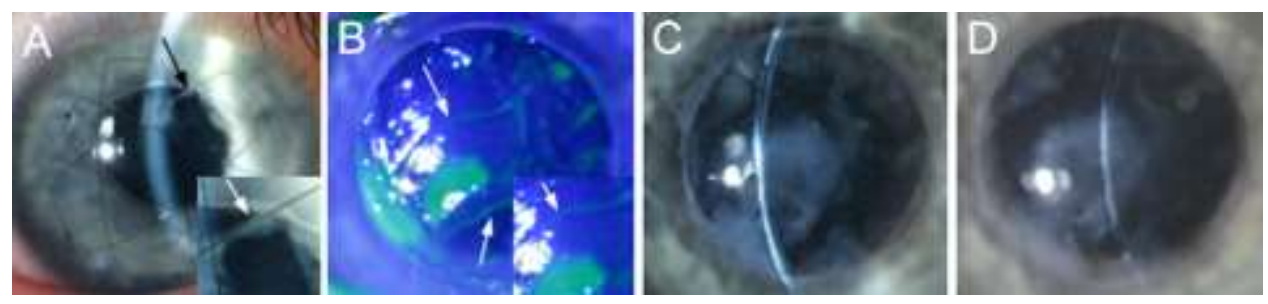

Figure 2. Example of postoperative corneal haze induced by delayed epithelial coverage of the implant. (A) Opaque areas along suture lines (arrow) were observed 3 weeks after surgery. (Inset) Subepithelial/stromal haze around suture (arrow). (B) After suture removal, fluorescein staining at 5 weeks shows a large area without epithelium, delineated by the position of sutures, magnified in inset (arrow). (C) Resulting discrete haze foci at 12 months and (D) 24 months after surgery, corresponding to the area of delayed epithelial coverage. Note that the cornea is transparent outside the haze areas. 


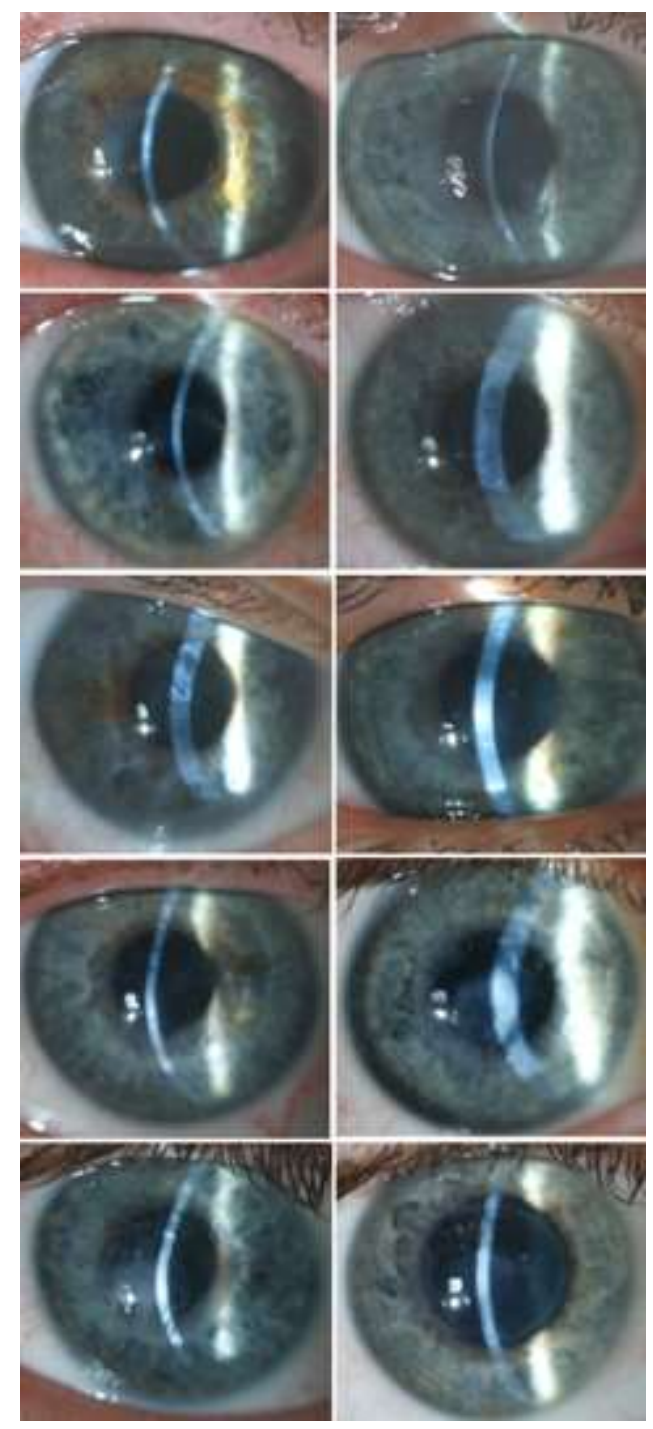

Fig.3. Slit lamp biomicroscope photographs of the ten eyes at 24 months after implantation with biosynthetic corneal substitutes. Implants were well-integrated into recipient corneas, with implant boundaries only barely visible. Focal areas of corneal haze were noted to varying degrees in eight patients at 24 months, while corneas were transparent outside these areas. 


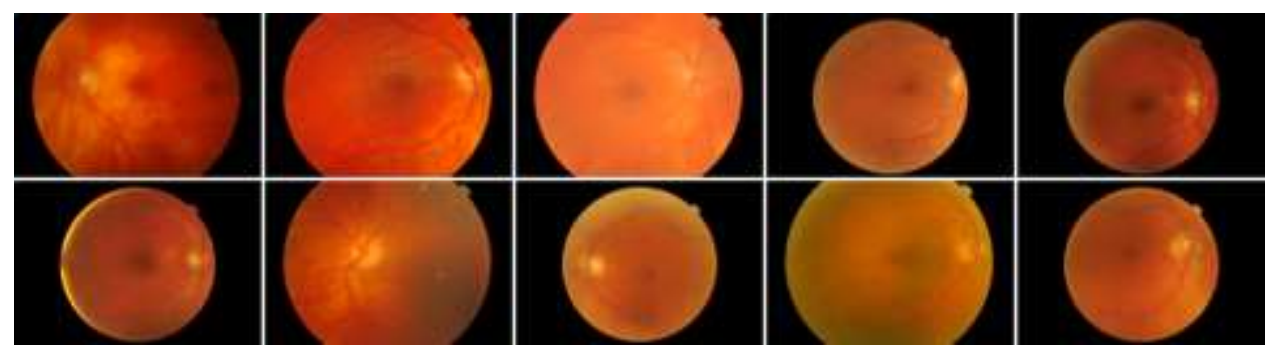

Fig. 4. Fundus images of the retina as photographed through the implanted biosynthetic corneas of all 10 patients at 24 months after surgery. Proper morphology of the retinal vessels was observed, demonstrating transparency of the implants even after regeneration of corneal tissue and nerves. 


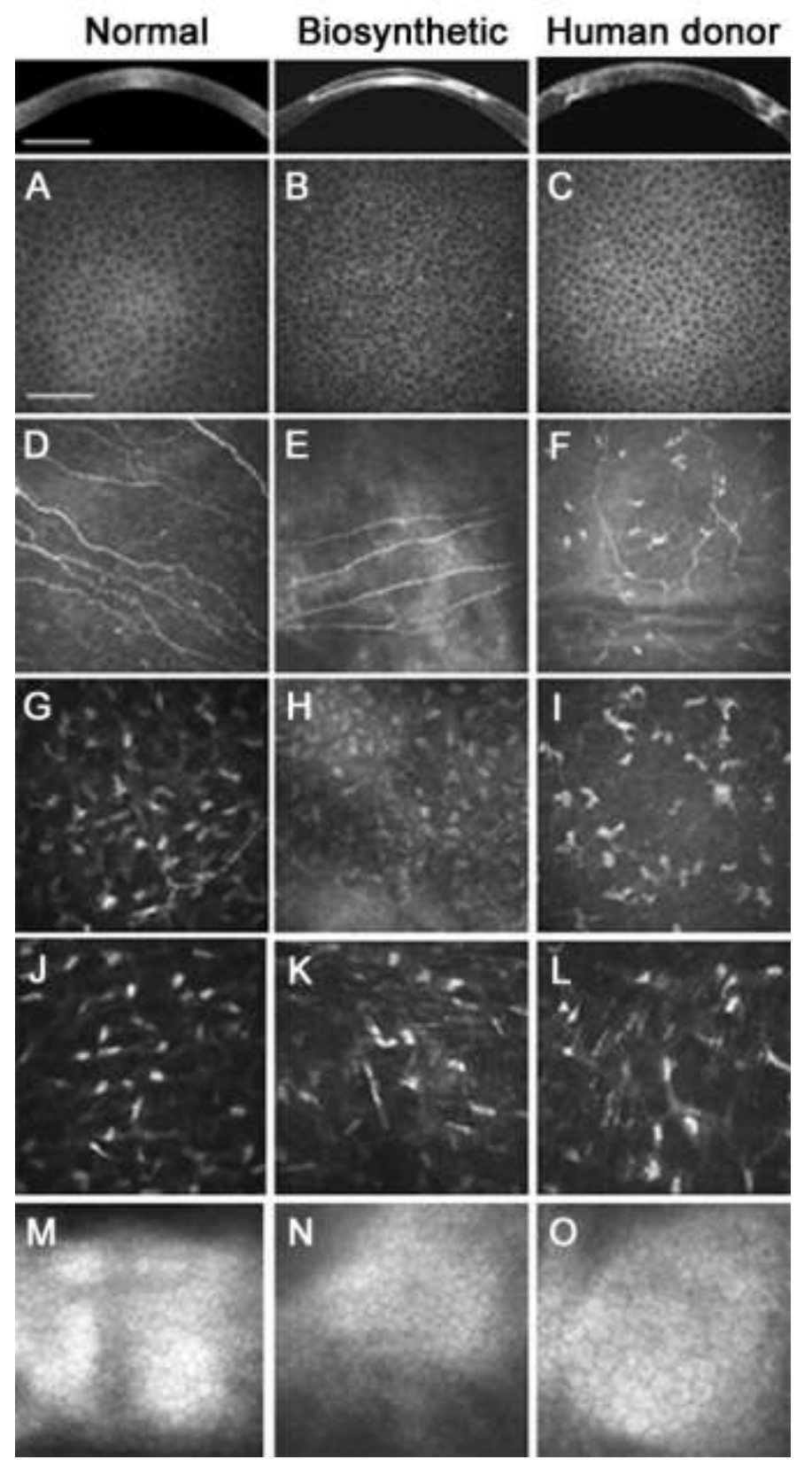

Fig. 5. A healthy, unoperated cornea (left column), compared to 24-month post-surgical regeneration in a representative biosynthetic corneal implant (middle column) and implanted human donor tissue (right column). (Top row) anterior segment optical coherence tomography (ASOCT) images of a healthy cornea, biosynthetic implant, and human donor transplant by penetrating keratoplasty. Areas of wound healing activity exhibit high reflectivity (white areas). (A through $\mathrm{O}$ ) In vivo confocal microscope 
images. Intact epithelium of the unoperated cornea (A), regenerated corneal epithelial cells on the implant surface (B), and regenerated epithelium of the penetrating graft (C). Regenerated nerves (E) at the sub-basal epithelium in an implanted cornea were parallel and morphologically similar to the normal cornea (D), while regenerated sub-basal nerves were also observed in a cornea transplanted with human donor tissue (F). Anterior stromal cell (keratocyte) nuclei $(\mathrm{G}-\mathrm{I})$, and posterior keratocytes $(\mathrm{J}-\mathrm{L})$ were present, with varying density, in all corneas. The endothelium $(\mathrm{M}-\mathrm{O})$ in all corneas exhibited a characteristic mosaic pattern. Scale bars: ASOCT, 1mm; in vivo confocal microscopy, $100 \mu \mathrm{m}$. 


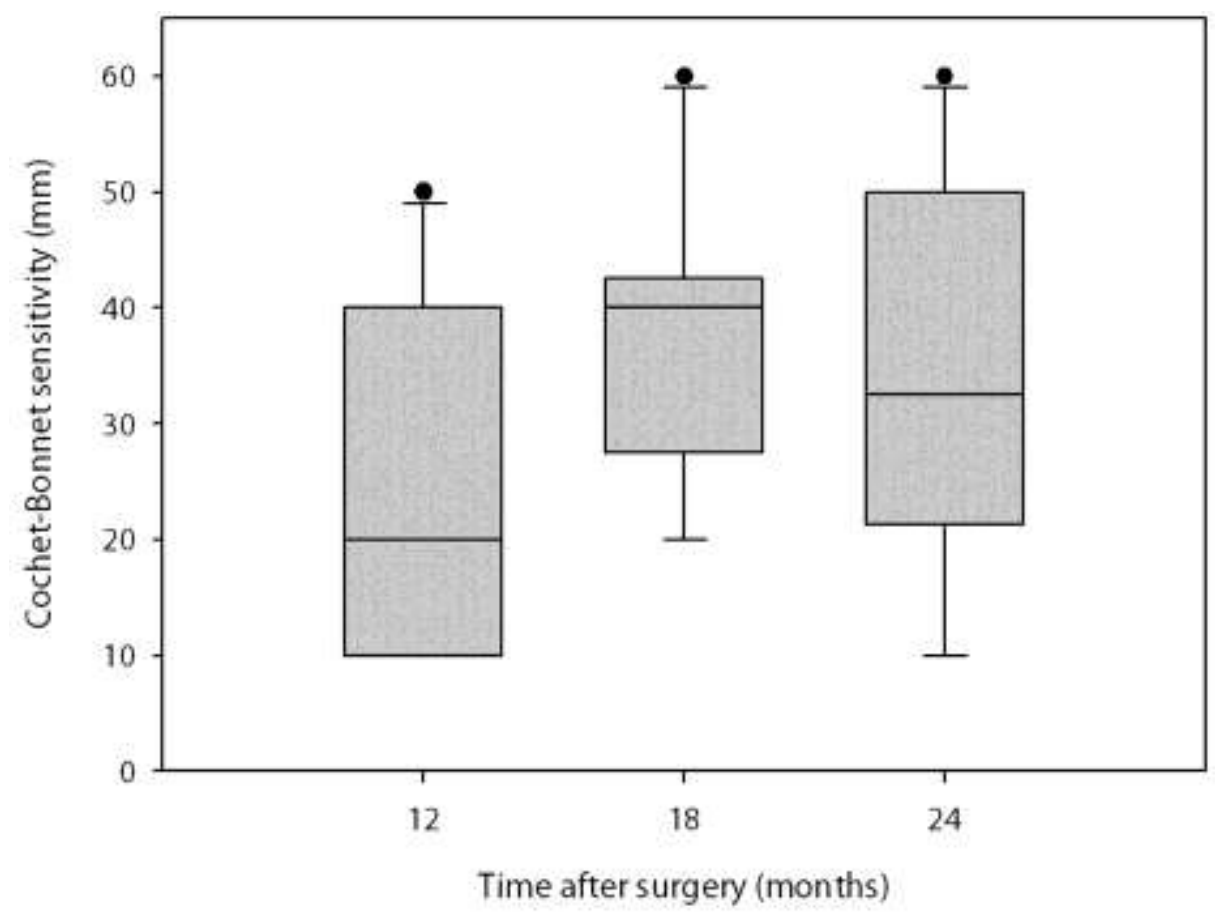

Fig. 6. Ocular surface sensitivity to mechanical touch stimulation in 10 patients as measured with Cochet-Bonnet aesthesiometry in implanted eyes. One cornea exhibited a return to normal sensitivity of $60 \mathrm{~mm}$ as early as 18 months. Box lines indicate mean, with whiskers indicating $5^{\text {th }}$ and $95^{\text {th }}$ percentile, and outliers are shown.

Table 1. Patient characteristics and summary of clinical results by patient. $\mathrm{CF}=$ counting fingers, SER $=$ spherical equivalent refraction, n.a. $=$ vision did not improve with spectacles.

Table 2. Central corneal subbasal nerve density after surgery as assessed by nerve tracing from in vivo confocal microscope images. 
Table 3. Mean visual acuity of patients implanted with biosynthetic and human donor corneas at 24 months after surgery. $\mathrm{SD}=$ standard deviation. 


\section{TABLES}

Table 1.

\begin{tabular}{|c|c|c|c|c|c|c|c|c|c|c|c|c|}
\hline \multirow{2}{*}{$\begin{array}{l}\text { Patient } \\
\text { No. }\end{array}$} & \multirow{2}{*}{$\begin{array}{l}\text { Age } \\
\text { (y) }\end{array}$} & \multirow{2}{*}{$\begin{array}{c}\text { Preoperative } \\
\text { Diagnosis }\end{array}$} & \multirow{2}{*}{$\begin{array}{l}\text { Sutures } \\
\text { removed } \\
\text { (weeks) }\end{array}$} & \multirow{2}{*}{$\begin{array}{l}\text { Time to full } \\
\text { epithelialization } \\
\text { (months) }\end{array}$} & \multicolumn{2}{|c|}{ BSCVA (decimal) } & \multicolumn{2}{|c|}{ Preoperative Refraction } & \multicolumn{2}{|c|}{$24 m$ Refraction } & \multicolumn{2}{|c|}{ 24m Tear Production } \\
\hline & & & & & Preoperative & $24 m$ & $\begin{array}{c}\text { SER } \\
\text { (dioptres) }\end{array}$ & $\begin{array}{c}\text { Astigmatism } \\
\text { (dioptres) }\end{array}$ & $\begin{array}{c}\text { SER } \\
\text { (dioptres) }\end{array}$ & $\begin{array}{c}\text { Astigmatism } \\
\text { (dioptres) }\end{array}$ & $\begin{array}{l}\text { Operated } \\
\text { (mm/5min) }\end{array}$ & $\begin{array}{c}\text { Fellow } \\
\text { (mm/5min) }\end{array}$ \\
\hline 1 & 75 & Keratoconus & 4 & 2 & CF $2 m$ & 0.1 & 2 & 6 & 3 & 6 & 31 & 21 \\
\hline 2 & 65 & Keratoconus & 6 & 5 & 0.2 & 0.2 & -1 & 6 & 1.75 & 2.5 & 26 & 24 \\
\hline 3 & 57 & Keratoconus & 5 & 3 & 0.2 & 0.1 & -1 & 6 & n.a. & n.a. & 23 & 6 \\
\hline 4 & 54 & Keratoconus & 4 & 3 & $<0.1$ & 0.4 & n.a. & n.a. & -7.5 & 3 & 35 & 31 \\
\hline 5 & 42 & Keratoconus & 6 & 2.5 & CF $1 \mathrm{~m}$ & 0.2 & n.a. & n.a. & 7 & 0 & 9 & 9 \\
\hline 7 & 35 & Keratoconus & 5 & 1.5 & 0.3 & 0.4 & -5.25 & 2.5 & -1.5 & 7 & 8 & 26 \\
\hline 8 & 30 & Keratoconus & 7 & 1 & 0.3 & 0.3 & -3.5 & 3 & 2.5 & 5 & 25 & 22 \\
\hline 9 & 26 & Keratoconus & 4 & 2.5 & $<0.1$ & 0.1 & -1 & 4 & 1.5 & 9 & 31 & 35 \\
\hline 10 & 18 & Central scar & 5 & 2 & 0.1 & 0.2 & n.a. & n.a. & -2.5 & 1 & 20 & 10 \\
\hline
\end{tabular}




\section{Table 2.}

\begin{tabular}{cc|c|c} 
Months after surgery & 12 & 18 & 24 \\
\cline { 2 - 4 } Number of patients with nerves & 5 & 8 & 9 \\
Density (mean \pm SD; $\left.\mu \mathrm{m} / \mathrm{mm}^{2}\right)$ & $654 \pm 751$ & $2115 \pm 2752$ & $4215 \pm 6048$ \\
$95 \%$ LOA $\left(\mu \mathrm{m} / \mathrm{mm}^{2}\right)$ & \pm 949 & \pm 1393 & \pm 2370 \\
\cline { 2 - 4 } $\begin{array}{c}\text { Nerve density, healthy subjects } \\
\left(\mu \mathrm{m} / \mathrm{mm}^{2}\right)\end{array}$ & $21600 \pm 5980 ; 30$ patients (40) \\
Nerve density, PK patients* & \multicolumn{3}{l}{$1830 \pm 3420 ; 42$ patients (40) } \\
\cline { 2 - 2 }$\left./ \mathrm{mm}^{2}\right)$ &
\end{tabular}

$\mathrm{SD}=$ standard deviation; $\mathrm{LOA}=$ limits of agreement for inter-observer measurements; $\mathrm{PK}=$ penetrating keratoplasty.

*measured from 1 month to 40 years after surgery (40) 
Table 3.

\begin{tabular}{cc|c|c} 
& \multicolumn{2}{c|}{ Biosynthetic } & Human donor \\
& 24m BSCVA & 24m BCLVA & 24m BSCVA (SCR) \\
\cline { 2 - 4 } No. of Patients & 10 & 10 & 60 \\
Decimal acuity & 0.18 & 0.48 & 0.46 \\
SD of acuity (lines) & 2.5 & 2.6 & 4.3 \\
Snellen acuity (feet) & $20 / 110$ & $20 / 42$ & $20 / 43$ \\
P-value* & $<0.001$ & 0.55 & - \\
*t-test with 24m PK data from the Swedish corneal register (SCR)
\end{tabular}


Figure S1

Slit lamp biomicroscopy showing the time course of epithelialization in Patient 3.
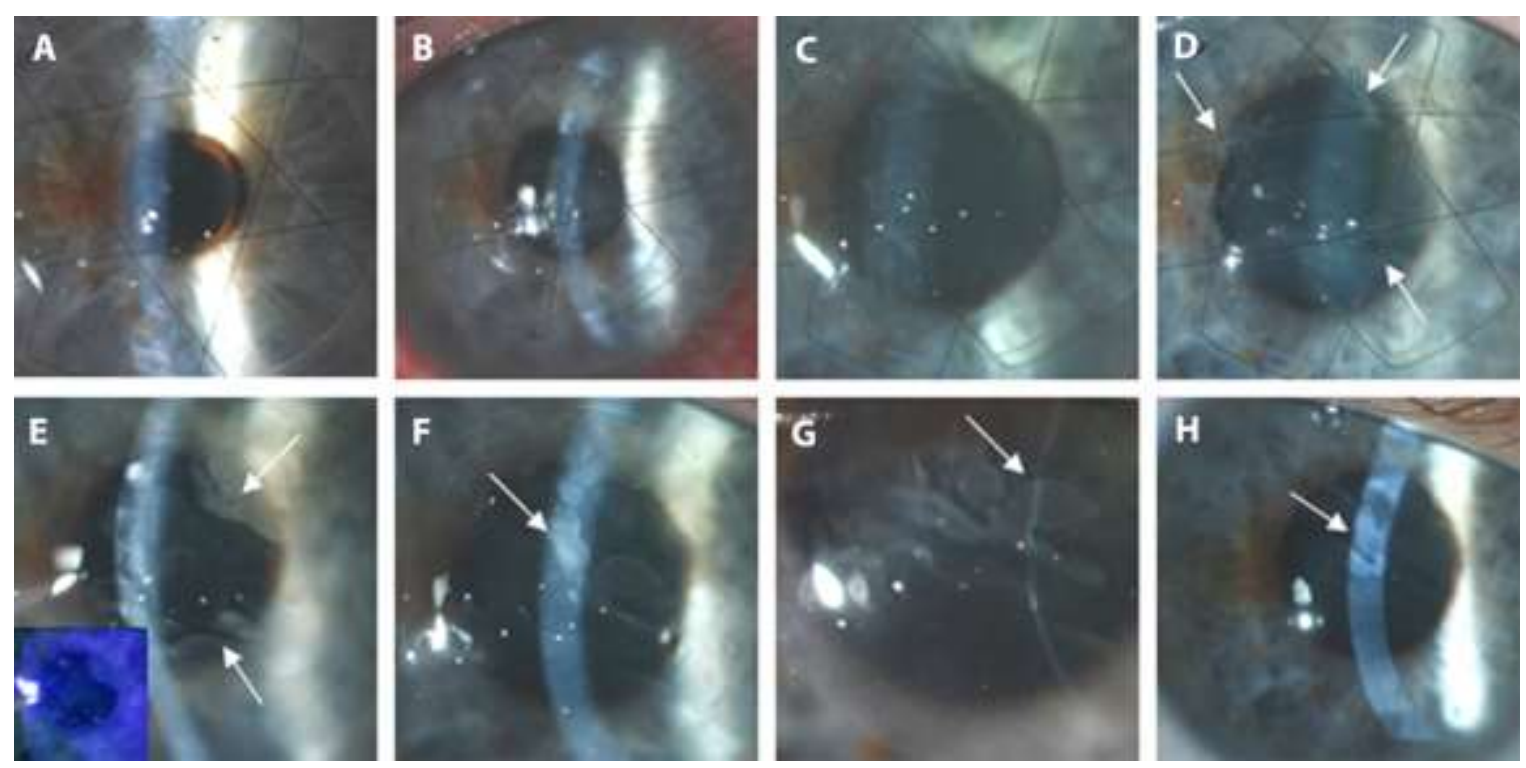

Time series of epithelialization of the implant in Patient 3. Slit lamp photographs were taken at the following times after surgery: (A) 1 day, (B) 1 week, (C) 2 weeks, (D) 1 month, (E) 2 months, (F) 3.5 months, (G) 6 months, (H) 24 months. (D) At 1 month, small hazy patches (arrows) appear at suture lines and within the implant. (E) By three weeks after suture removal, epithelialization is complete (cornea does not stain with fluorescein, inset), although hazy patches are evident in the stroma (arrows). ( $\mathbf{F}$ through $\mathbf{H}$ ) hazy patches (arrows) persist in the stroma to 24 months. 
Figure S2

Slit lamp biomicroscopy showing the time course of epithelialization in Patient 6.
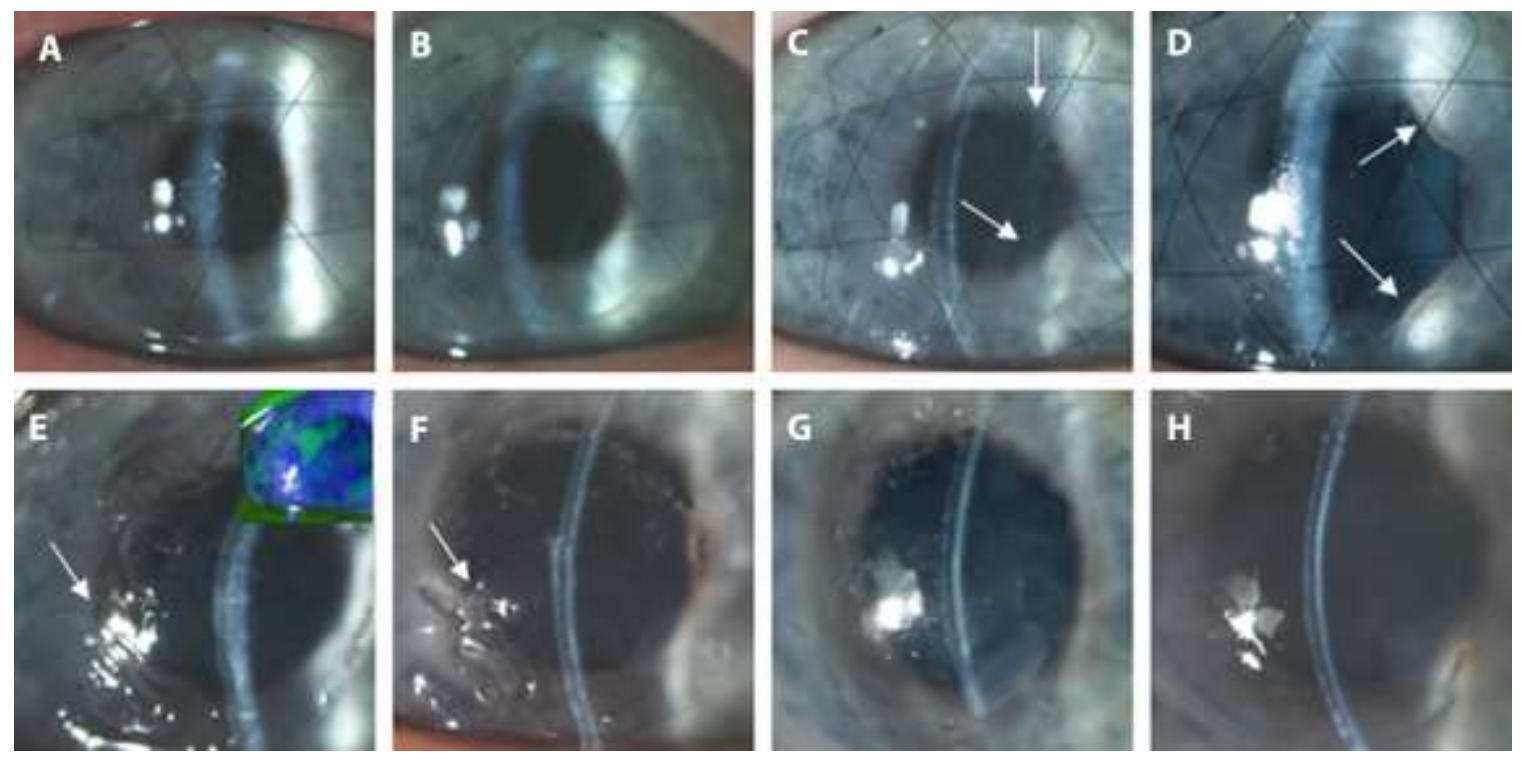

Time series of epithelialization of the implant in Patient 6 . Slit lamp photographs were taken at the following times after surgery: (A) 1 day, (B) 1 week, (C) 2 weeks, (D) 1 month, (E) 2 months, (F) 3.5 months, (G) 6 months, (H) 24 months. (C) At 2 weeks, the edges of an epithelial sheet (arrows) advanced towards the implant center. (D) At 1 month, epithelial advancement (arrows) had not progressed. (E) Four weeks after suture removal, epithelialization was incomplete (patchy staining with fluorescein - green, inset) and some surface irregularity was evident (arrow). (F) Surface irregularity persisted (arrow, and uneven projection of slit). (G, H) The implant surface became more regular after six months. 
Figure S3

Stromal cell migration into the biosynthetic implant at 24 months in four patients.
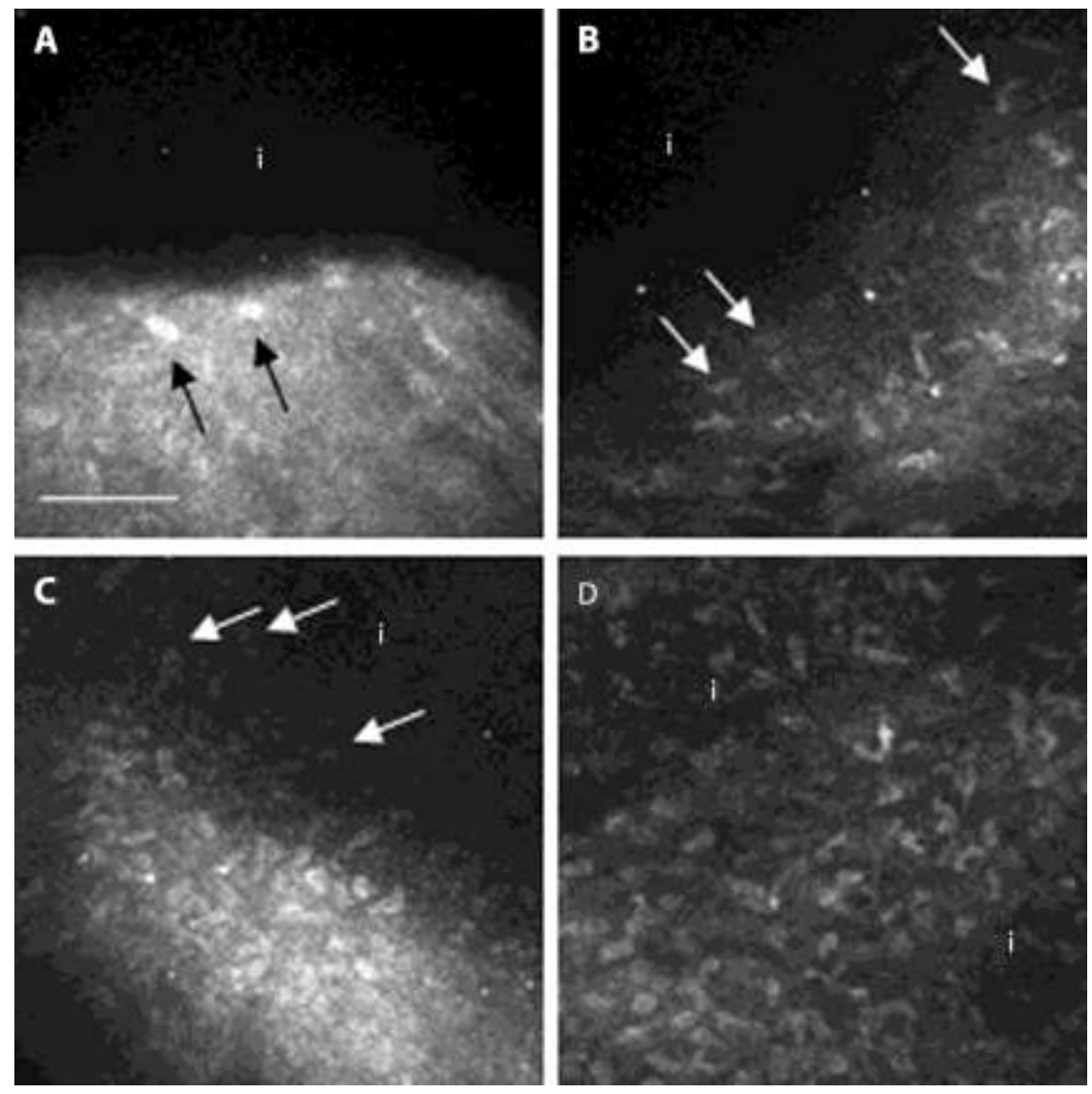

Variability in stromal cell migration into the biosynthetic implant at 24 months in four patients, as recorded by in vivo confocal microscopy. (A) Fibrous, reflective scar tissue at the posterior implant interface contains host cells (arrows) that have not invaded the implant ( $\mathrm{i}$ ), resulting in a distinct implant-to-host interface. (B) A few host cells (arrows) have migrated into the implant. (C) Migration of numerous cells (arrows) into the implant. (D) Substantial repopulation of the implant by host stromal cells results in a smooth transition from host tissue to implant. Images were taken from the central cornea. Scale bar, $100 \mu \mathrm{m}$. 